\title{
A CNN-based scheme for COVID-19 detection with emergency services provisions using an optimal path planning
}

\author{
Ahmed Barnawi $^{1} \cdot$ Prateek Chhikara $^{2} \cdot$ Rajkumar Tekchandani $^{2} \cdot$ Neeraj Kumar $^{1} \cdot$ Mehrez Boulares $^{1}$
}

Received: 28 September 2020 / Accepted: 8 July 2021

(c) The Author(s), under exclusive licence to Springer-Verlag GmbH Germany, part of Springer Nature 2021

\begin{abstract}
Unmanned Air Vehicles (UAVs) are becoming popular in real-world scenarios due to current advances in sensor technology and hardware platform development. The applications of UAVs in the medical field are broad and may be shared worldwide. With the recent outbreak of COVID-19, fast diagnostic testing has become one of the challenges due to the lack of test kits. UAVs can help in tackling the COVID-19 by delivering medication to the hospital on time. In this paper, to detect the number of COVID-19 cases in a hospital, we propose a deep convolution neural architecture using transfer learning, classifying the patient into three categories as COVID-19 (positive) and normal (negative), and pneumonia based on given X-ray images. The proposed deep-learning architecture is compared with state-of-the-art models. The results show that the proposed model provides an accuracy of $94.92 \%$. Further to offer time-bounded services to COVID-19 patients, we have proposed a scheme for delivering emergency kits to the hospitals in need using an optimal path planning approach for UAVs in the network.
\end{abstract}

Keywords Computer vision $\cdot$ COVID-19 $\cdot$ Path planning $\cdot$ Transfer learning $\cdot$ Unmanned Aerial Vehicle

\section{Introduction}

Unmanned Aerial Vehicles (UAVs), popularly known as drones, were used mainly for military purposes. Currently, their use cases have increased to achieve civilian tasks like search and rescue operations [1], indoor navigation [2], delivering goods [3], monitoring traffic flow, and weather $[4,5]$. Nowadays, they are used by artists for aerial photography as well. In the agriculture field, UAVs are used to spray fertilizers and track crop growth patterns [3]. Recently,

Neeraj Kumar

neeraj.kumar.in@ieee.org

Ahmed Barnawi

ambarnawi@kau.edu.sa

Prateek Chhikara

pchhikara_be16@thapar.edu

Rajkumar Tekchandani

rtekchandani@thapar.edu

Mehrez Boulares

mboulares@kau.edu.sa

1 Department of Information Technology, King Abdulaziz University, Jeddah, Saudi Arabia

2 Department of Computer Science and Engineering, Thapar Institute of Engineering and Technology, Patiala, India
UAVs in healthcare logistics have shown great success by improving medical deliveries and services. Figure 1 shows the expansion of the medical UAV market all over the world. The speculations are that the UAV market will be around $\$ 400$ million by 2025 . UAVs can perform deliveries such as large-scale blood, long-tail medicines, medical samples, and even organs. With the advancement in technology, the modern healthcare system serves some of the most prominent human intelligence accomplishments to enhance people's lives. The rural and underdeveloped areas of the world still lack proper healthcare facilities. Filling these gaps has realized a new necessity during the current pandemic, which has shown the interconnection between our health outcomes [6]. Regardless of the location, UAVs can give on-time supplies of necessary medical items. It is challenging to store cold-chain products such as platelets or blood in a place for a long time, so UAVs can deliver these products when there is a demand for the same within a stipulated time.

\subsection{Drones used in medical field}

Previous works prove that drones can safely deliver medicines from the medical health center to those in need. Essential medication such as Insulin is delivered using UAVs in the past. The most significant advantage that UAVs provide 


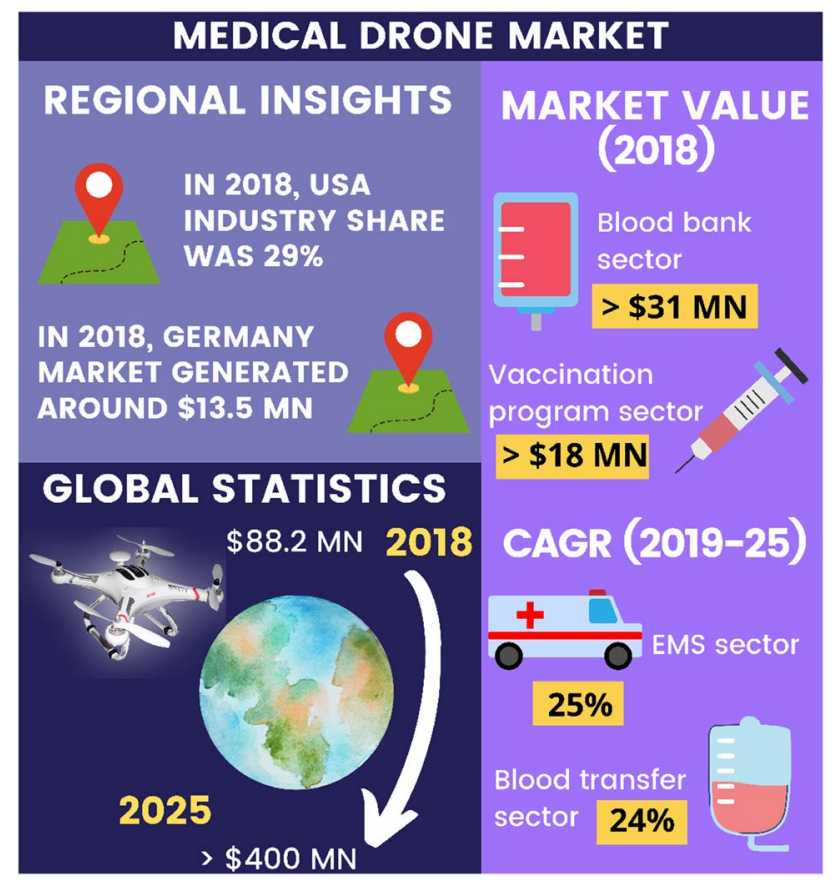

Fig. 1 Medical Drones Market size was valued at USD 88.2 million in 2018 and is expected to witness $24.7 \%$ CAGR from 2019 to 2025 [7]

is the delivery speed. UAVs for medicine delivery purposes will avoid the production of large stocks of opioids in private houses. When breakthrough pain is expressed by a patient undergoing palliative care, quick delivery of the required medicine can be done with the necessary dose for the pain control and per the patient's needs. Dr. Joseph Scalea (a transplant surgeon) was frustrated by the slow speed and high cost of commercial flights, so he explored faster ways for delivering kidneys, livers, and other organs that can quickly deteriorate. In April 2019, Dr. Scalea from the University of Maryland Medical Center delivered a kidney that was successfully transplanted into a patient undergoing a severe nephrological condition; this was the first-ever UAV delivery of a human organ in history [8]. Table 1 shows the medicines that can be delivered using UAVs in different locations [9].

\subsubsection{Drones used in the medical field during COVID-19}

Novel coronavirus disease (known as COVID-19) has significantly impacted the world, with $30+$ million confirmed cases and a million deaths globally. Early and precise diagnosis of COVID-19 is of high importance for restraining the spread of the disease and to reduce death [10]. In the current state of the pandemic COVID-19, this usage of UAVs can prove to help reduce the load on the hospitals and health care staff. UAVs can be used for the fast delivery of medical kits and supplies (1) from one medical facility to another, (2) from a central base medical station (warehouse of medicines) to hospitals for the COVID-19 patients [11]. For example, in China, a UAV was used to move medical supplies from the disease control center in Xinchang County to the People's Hospital in Xinchang County without infecting humans [8]. To fight the COVID-19 pandemic in India, a Hyderabad-based startup headed by a team of Indian Institute of Technology (IIT) alumni launched a complete series of UAVs.

\subsection{Motivation}

The government of Punjab, India, provided 50,000 free-ofcost COVID care kits (FATEH kits) comprising Oximeter, Digital Thermometer, Face Masks, and necessary medication to active patients in hospitals and homes to ensure better care for them. The move is part of the broader state government endeavor to ensure that all patients get complete medical care at their doorstep, thus facilitating their early and complete recovery from the disease [12]. This can be further extended using UAV facilities that replace manual delivery of kits, thus reducing the human intervention and provides time-bounded services. In most cases, the use of drones during the COVID-19 pandemic has served as an important asset that reduces the virus infections spread in delivering automated external defibrillators
Table 1 Medicines that can be delivered by the UAVs

\begin{tabular}{llll}
\hline Location & Clinical need & Product example & Benefit \\
\hline Out of clinic & Allergic reaction & Epi pen & Rapid transfer \\
& Cardiac arrest & Atropine & Rapid transfer \\
& Asthma attack & Salbutamol Nebulizer & Rapid transfer \\
& Clotting agent & Live leaches & Centralized supply \\
& Dispensing controlled drugs & Morphine & Control \\
In clinic & Specialized diagnostic test & Blood, tissue, etc. & Centralized supply \\
& Nuclear imaging & Half-life radionucleotide & Rapid transfer \\
& Burn debriding & Live maggots & Centralized supply \\
& Chemotherapy & Half-life radionucleotide & Rapid transfer \\
In field & Disease outbreak & Vaccine & Remote delivery \\
& Snake bite & Antivenom/antidote & Remote delivery \\
\hline
\end{tabular}


(AEDs) and personal protective equipment (gloves, facemasks, etc.) at emergency sites and health facilities in various inaccessible areas with minimal human intervention, causing less virus spread [13]. Hence, a path planning technique using UAVs should be devised which takes the fuel onboard and the amount of medication left under consideration. The UAV's objective is to cover the maximum number of hospital facilities in a given search space with fuel and within the stipulated time limit. This paper proposes a scheme that offers the shortest path between the source warehouse and the destination warehouse considering all the mentioned constraints.

Currently, for the detection of COVID-19 in a patient, Reverse Transcription Polymerase Chain Reaction (RTPCR) is used as the standard method [14]. This test comprises the detection of viral nucleic acid from sputum or nasopharyngeal swab. The limitations of this test are: (i) this test needs special supplies, which are not accessible at every place, (2) this test consumes time and has a comparatively low sensitivity or true-positive rate, (3) this test is complex and most of the times uncomfortable for the patients. Hence, due to the mentioned concerns, alternative diagnostic methods should be proposed that address these concerns. Chest X-ray images have life-saving importance for both patients and doctors in countries with a high population and cannot purchase laboratory kits for testing. Therefore, devising an automated tool for detecting COVID-19 becomes even more vital [15]. The Fleischner Society recommends that medical practitioners use digital chest X-rays and CT scans to manage COVID19. In the end, the choice of imaging modality is left to the judgment of clinical teams at the point of care, accounting for the differing attributes of chest radiography and CT, local resources, and expertise [16]. In addition, detecting infected patients in an early stage is a vital step in fighting COVID-19, and detecting this disease from radiography and radiology images is possibly one of the fastest ways to diagnose the patients [17]. Convolutional Neural Network (CNN) has shown success in numerous computer vision, speech recognition, and robotic control applications. With the availability of massive datasets and powerful computational power, CNN can extract high- and low-level features from the dataset, leading to the performance equal to humans in various applications. Furthermore, efficient $\mathrm{CNN}$ architecture gives an accurate prediction and is computationally efficient as well. Hence, researchers selected CNNs as an applied AI diagnosis algorithm for COVID19. The advantages of using this approach are efficiency and learning through backpropagation. In the proposed work, we have created an autonomous CNN model that takes a digital chest $\mathrm{X}$-ray image as an input and classifies the image into three categories, namely COVID-19 (positive), normal (negative), and pneumonia.

\subsection{Contributions}

The contributions are summarized as follows:

(1) A pre-processing step is introduced using various statistical image enhancement techniques (such as removing blur and noise, sharpness, contrast correction, etc.) to enhance the illumination in the digital X-ray images and improve overall image quality before feeding it to the neural network.

(2) A deep convolutional neural network using transfer learning is proposed, classifying a given chest X-ray input image into COVID-19, normal, or pneumonia class. Moreover, the proposed model is compared with the existing state-of-the-art models.

(3) An optimal path-planning scheme is designed to deliver emergency medical kits to the hospitals in need with lesser cost and following constraints regarding available fuel (battery life), available medicine, and specified time limit.

Following, the related work is discussed in Sect. 2. The preprocessing step and the proposed CNN model, and an optimal path planning scheme for UAV in delivering the medical kit are explained in Sect. 3. The implementation details and the quantitative and qualitative results are described in Sect. 4 . Section 5 presents the conclusion and discusses the future work.

\section{Related work}

This section explores the existing work in the literature aligning with our proposed approach in the paper.

\subsection{Approaches applied to UAV network mobility}

In this section, we present the research work regarding the mobility of UAVs in a network. Shi et al. [18] proposed a drone-supported radio access network scheme in which drone cells are leveraged to deliver data connecting base stations and users. They first analyzed the user coverage and the D2B backhaul link features of drone cells. They formulated a 3-D drone-cell deployment problem to maximize the user coverage while keeping D2B link quality. Sharma et al. [19] used drones using LoRaWAN gateway and introduced a communication approach based on the energy consumption, area stress, and other resilient factors that avail in the effective localization, enhanced coverage, and energy effective monitoring with lesser overheads and redundancy, with almost negligible isolations. Shakhatreh et al. [20] used a single UAV to implement a wireless coverage scheme for indoor users inside a high-rise building under disaster 
conditions such as earthquakes or floods, in the scenarios when cellular networks are not working. Ghamry et al. [21] discussed forest fires fighting application using a swarm of UAV. The fire-fighting UAVs receive an appropriate message, and based on the message, the team undertakes the task. To minimize the distance traveled between UAV's initial position and its assigned fire spot, they proposed an algorithm that allocates UAVs to different fire spots according to their relative distances. Berger et al. [22] used a team of heterogeneous UAVs equipped with LIDAR and other sensors for scanning an outdoor area and create a 3D map for the same. They also minimized the exploration time of the UAVs. You et al. [23] proposed a novel network formation consisting of nodes from different networks maneuvered by UAVs to provide continuous and robust connectivity. The model uses the features of a biological perspective of genomes and creates a delayed receptive network. The model is tested both in real-time and simulation environments, and the result shows the efficiency in data delivery, fewer overheads, and lesser latency with a high convergence rate.

\subsection{CNN models for detecting COVID-19 in chest $\mathrm{X}$-ray images}

Ismael et al. [24] used deep-learning-based approaches, namely deep feature extraction. For the extraction of the features, they used variations of ResNet and VGG models, and for the classification of the features, the Support Vector Machines (SVM) classifier is used. Jain et al. [25] used a deep learningbased CNN model and compared its performance with other state-of-the-art models such as InceptionV3, Xception, and ResNeXt models. Ibrahim et al. [26] created a classifier that can classify a wider range of chest X-ray images belonging to the COVID-19 class itself, such as bacterial pneumonia, nonCOVID-19 viral pneumonia, and the normal chest X-ray scans, by using a deep-learning approach based on a pre-trained AlexNet model. Wang et al. [27] proposed a computer-aided detection (CAD) system using computer vision to differentiate COVID-19 from community-acquired pneumonia (CAP) on chest X-rays. Their approach was divided into two steps as the Discrimination step and the Localization step. Mahmud et al. [28] proposed a CNN-based architecture (CovXNet), that uses depthwise convolution operation with varying dilation rates for the extraction of diversified features from chest $\mathrm{X}$-rays. Different forms of CovXNets models are designed and trained with X-ray images of different resolutions, and the model was optimized using a stacking algorithm. Hassantabar et al. [29] used three deep learning-based methods for detecting and diagnosing COVID-19 patients using chest $\mathrm{X}$-ray. They presented two algorithms, a deep neural network applied to the fractal feature of images and a CNN applied on the chest X-rays. Zabirul et al. [30] combined the CNN with a Long Short-Term Memory (LSTM) model for detecting the
COVID-19 in chest X-ray images. They used CNN as a feature extractor, and for the detection, LSTM is used. Shibly et al. [31] proposed a deep neural architecture using the approach of a faster R-CNN model to detect COVID-19 patients from chest X-ray images. Abraham et al. [32] discussed the effectiveness of multi-CNN architecture, a combination of different pre-trained CNNs. Their extracted features using a multi-CNN architecture with a correlation-based feature selection method and finally predicting COVID-19 in the chest X-ray were done using the Bayesian classifier. Vrbancic et al. [33] proposed an adaptive classification method that can identify COVID-19 patients based on a chest X-rays. They used a grey wolf optimizer algorithm to optimize hyper-parameter within the transfer learning tuning of a CNN. Fathurahman et al. [34] compared the performance of a 1-D convolutional filter to classify pneumonia with various state-of-the-art CNN architectures to deliver the best accuracy. Toraman et al. [35] proposed an artificial neural network (CapsNet) with capsule networks for the detection of COVID-19 disease using chest X-rays. The proposed approach provides fast and accurate diagnostics for COVID-19 diseases with binary classification (COVID and normal) and multi-class classification (COVID, normal, and pneumonia).

The CNN models are created to detect COVID-19 using a chest X-ray as an input in related work. In these models, the chest X-ray images are directly used as input without any pre-processing step. Hence, there was a scope for improvement, which can be achieved by creating a proper pipeline containing the image enhancement approaches. If the input is pre-processed well, then the model will learn better and achieve more accuracy. The desired features of images can be extracted using various image enhancement techniques, as training of deep-learning models relies on the input features. There should be a scheme that can deliver the medicines to the desired location in the stipulated time. The current work mainly uses the Dijkstra algorithm and other graph algorithms such as BFS or DFS to find the best suitable path from the source to the destination. The problem with these approaches is that they only focus on the distance between the two nodes, which is not the only factor considered in real-world scenarios. Hence, other factors such as the fuel in hand and the amount of material that can be delivered to the next node (as if there is no material left to deliver to the upcoming node, then there is no need to visit that node, it will simply increase the time), etc., should also be considered.

\section{Proposed work}

We have proposed a CNN architecture for the detection of COVID-19 in a patient using chest X-ray images. Before feeding the raw chest X-ray images directly to the classifier, 
the images are pre-processed using Digital Image Processing techniques to enhance the image's import features. Data Augmentation is also done only on the training set to increase the training data, thus handling the classifier's under-fitting. After successfully creating and testing the classifier, the classifier is deployed in hospitals. With the proposed classifier's help, hospitals can tell the number of cases on time and ask for emergency medications. Therefore, UAVs will come into the picture for delivering emergency medical kits to the hospitals in need on time. For efficient delivery, we devise an optimal path-planning scheme that chooses the path that consumes less time and considers the battery life and the medicine available with the UAV. The proposed approach is divided into two parts. In Sect. 4 (A), we will discuss the proposed $\mathrm{CNN}$ architecture, and in Sect. 4 (B), we will discuss the efficient scheme for UAV path planning.

\subsection{Transfer learning}

The CNN model has an outstanding performance in computer vision applications. Training a complete $\mathrm{CNN}$ model typically needs a large dataset; otherwise, the model will overfit, and the over-fitting will rise to a less generalized model [36]. In many fields of medical imaging, the image dataset is insufficient. Hence, training a complete CNN on fewer data is challenging. To solve this difficulty, transfer learning is used. Transfer learning works on the principle of fine-tuning, which means change the parameters of a pretrained CNN model [37]. There are many approaches for fine-tuning parameters. The standard methods are fine-tuning all convolutional and fully connected layers or freezing some layers, and fine-tune the remaining layers.

\subsection{Calculation of number of COVID-19 cases using CNN}

This is divided into two steps; we will discuss the pipeline used to enhance the chest X-ray images using DIP in the first step. In the second step, we will discuss the proposed architecture and approach for training the model.

\subsubsection{Pre-processing of dataset}

The largest data source in the healthcare field is in the form of images (such as MRI, CT scans, X-rays, ultrasound, PET, etc.), and one of the most complex sources to interpret. Clinicians today rely mainly on medical image analysis performed by radiologists and sometimes examine scans themselves [38]. Even patients use imaging technology to monitor treatment. These medical images can be improved with the use of AI and DIP. The best image enhancement approach is not achieved using a single operation, but multiple techniques are used to achieve better results. In the proposed work, a pipeline is created to enhance the quality of chest X-ray images using the image enhancement methods as shown in Fig. 2. The input image is shown in Fig. 2 (1). First, we apply the Laplacian filter (Laplacian filter outputs sharp edges and then subtraction is performed to get the enhanced image.) [39] in Fig. 2 (1), and the output image Fig. 2 (5) is subtracted from the original image to get a sharpened image Fig. 2 (4). Then the Sobel filter [40] is applied on Fig. 2 (1) followed by Gaussian Blur [41] to get the output image as Fig. 2 (3). Now, we apply an unsharp masking approach to our original input image. In the unsharp masking approach, we subtract a smoothed version of an image from the original image. The process is achieved in the following three steps:

(1) Blur the original image: the blurred image is Fig. 2 (3),

(2) Creating a mask: the product of Fig. 2 (3) and (4), acts as a mask shown in Fig. 2 (6),

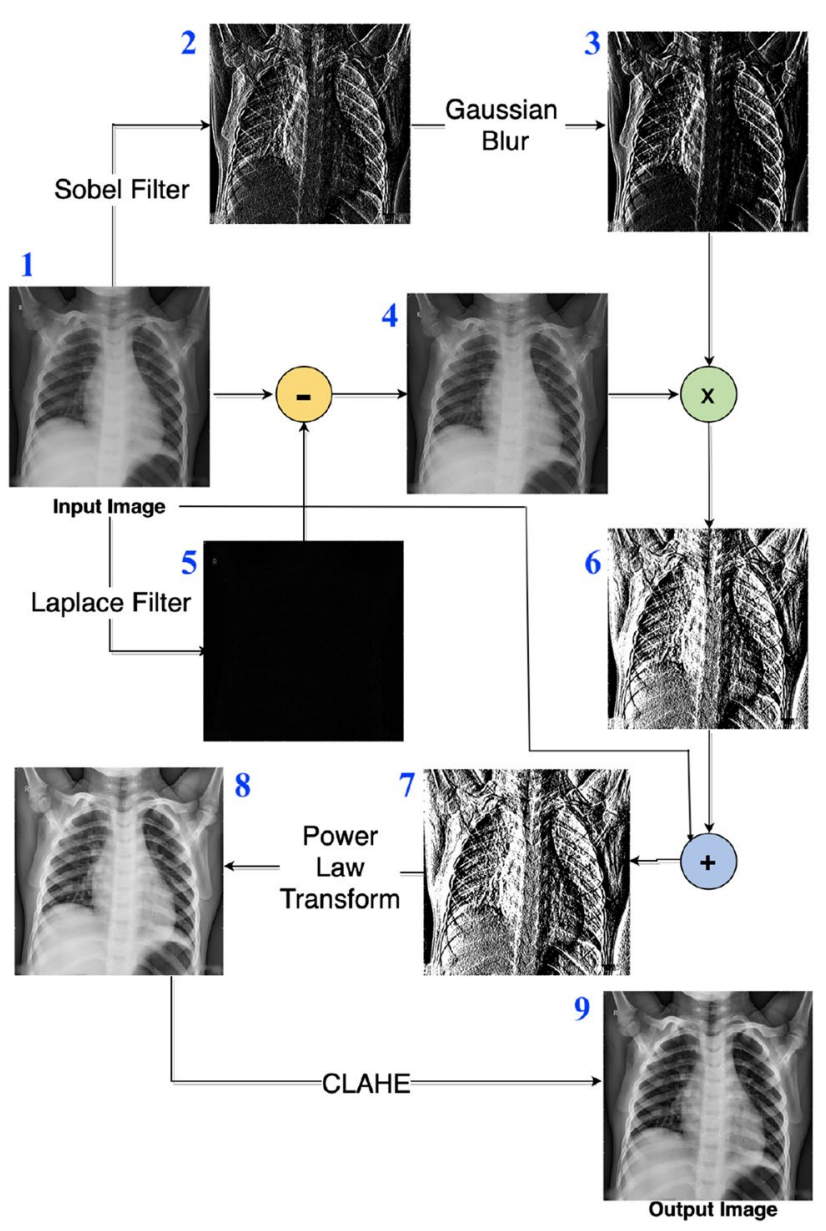

Fig. 2 Pre-processing of the dataset using image enhancement techniques before feeding to the $\mathrm{CNN}$ architectures 
(3) Add the mask to the original: the mask is added to the original input image, and the result is the sharpened image, shown in Fig. 2 (7).

A gamma transformation is applied to this sharpened image (Fig. 2 (7)), and finally, to increase the contrast of the image CLAHE [42] is used. The resultant enhanced image is shown in Fig. 2 (9). The proposed model without pre-processing step showed an accuracy of $93.96 \%$, while after applying the pre-processing step, the accuracy increased to $94.92 \%$.

\subsubsection{Model training}

The model is trained using Inception $\mathrm{V} 3$ architecture as a backbone. The final output layer of 1000 nodes is removed and replaced with one flatten layer followed by two fully connected dense layers containing ' 128 ' and ' 48 ' nodes. Finally, one more dense layer is added containing three nodes (equal to the output class number). The dropout layer is also used after each of the dense layers except the last one. The proposed architecture is shown in Fig. 3. The model is trained on $75 \%$ of the whole dataset. The dataset was resized to $224 \times 224 \times$ three before feeding it to the network. Data augmentation is used while training the classifier. While training, the input images are rotated with a limit of $10^{\circ}$; the images were flipped horizontally and zoomed with a zoom range of 0.2 . A batch size of ' 64 ' is used while training the model for ' 50 ' epochs. The learning rate is initialized with 0.001 , and it will decrease by $10 \%$ if there is no progress in the accuracy for ' 10 ' epochs. To converge faster, the 'adam' optimizer is used while monitoring the training accuracy. Categorical cross-entropy is used as the loss function for the training purpose.

\subsection{Scheme for finding the optimal route for medical kit delivery}

Figure 4 shows the hospital, drone, medicine refill network. The hospitals are denoted as $\mathrm{H} 1, \mathrm{H} 2$, and $\mathrm{H} 3 ; \mathrm{S}$ is the starting point where the drone will take off carrying the emergency medical kit; $T$ is the drone's landing point. Both starting $(S)$ and the target point $(T)$ can work interchangeably. At both $S$ and $T$, the drone can refill the medicine, and services to the drones are also provided there, like calibrations and testing.

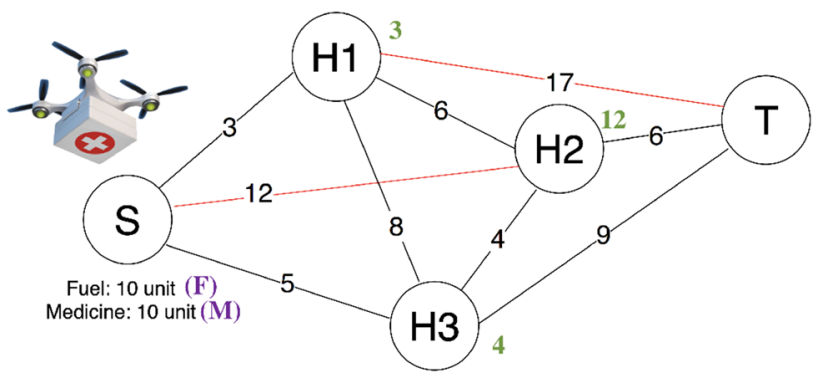

Fig. 4 Graph showing the connection of the hospital, drone stations, and medicine refill station

Objective: The drone will start at node $(S)$, having the full battery charged $(F)$ and with $(M)$ amount of medication. The drone has to reach the target $(T)$ with a minimum cost, such that it covers as many hospitals as it can, keeping the battery and medicine available in consideration.

Given data: All the hospitals are interconnected and they are connected to ' $S$ ' and ' $T$ ' as well. Therefore, if there are ' $n$ ' hospitals, then the degree of a hospital node will be equal to $[(n-1)+2]$, and the degree of the ' $S$ ' and ' $T$ ' node will always be equal to ' $n$ '. The edges in the graph are the distance between the nodes. Each hospital node has some fixed medicine requirement, and the drone will deliver that amount only. Charging stations are placed at every hospital, which gives the privilege to the drones to charge themselves.

Proposed scheme: The number of medicines required by a hospital will be proportional to the number of cases identified by our proposed CNN model. If the drone has no medicine left, then in place to go to the next hospital emptyhanded, it will go straight to the target ( $T$ ). If a hospital has zero medicine requirements, then that hospital will not be considered part of the network. If the distance between the two nodes in the network is more significant than what a drone can travel with a full charge battery, then that path should be removed from the network.

Case study: In Fig. 4, the drone has the battery capacity $(F)$ of 10 units (directly proportional to the distance that the drone can cover), and the medicine capacity $(M)$ of 10 units as well. The edges having a length greater than ten units (marked as red) are removed because the drone's max distance can travel ten units. Each hospital has some medicine requirements, as shown in green text. Now, we will find all the paths in the network satisfying our objective. All the
Fig. 3 Proposed CNN architecture using InceptionV3 as a backbone

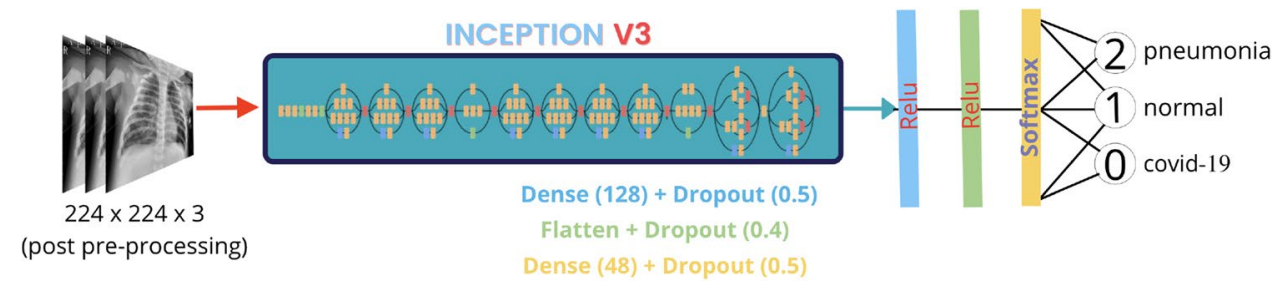


paths that the drone can take without failing are shown in Table 2, with their associated cost and the number of hospitals that the drone can visit. Out of the seven paths, path number '2' (Fig. 5a), 4 (Fig. 5b), and '6' (Figure 5c) are considered because all these three paths are covering all the three hospitals. Now we have three candidate paths, and we have to choose the optimal out of them. In the case of path number ' 4 ', the drone will first travel to the hospital (H1), which requires ' 3 ' units of medicine. After visiting the $\mathrm{H} 1$, the drone will now have ' 7 ' units of medicine. Now, the drone will visit $\mathrm{H} 2$, which has a requirement of ' 12 ' units. After visiting this node, the drone will be left with zero medicine, and hence it will not travel to H3. Therefore, we will discard this path for now. We will now consider paths ' 2 ' and ' 6 '. With both these paths, the drone can travel to all three hospitals with at least some medicine in hand. Hence, out of these two paths, we will consider path number ' 2 ' to be the optimal path because it has ' 21 ' cost value associated with it, and in path number ' 6 ', the cost value is ' 25 '. After using the proposed optimal pathfinding scheme, the drone can traverse to as many hospitals as possible with minimal cost and keep the constraints in mind.

\section{Experimental results and discussion}

\subsection{Dataset}

A public database is used, which is generated by collecting images from several image data sources [43]. All the images are in Portable Network Graphics (PNG) file format, with a resolution of $1024 \times 1024$ pixels and $256 \times$ 256 pixels, which can easily be converted to $224 \times 224$ or $227 \times 227$ pixels for input to popular CNN networks. The dataset consists of three classes, namely COVID-19, normal, and pneumonia. The COVID-19 class is labeled as ' 0 ' that contains 219 images, the normal class is labeled as ' 1 ' containing 1341 images, and the pneumonia class is labeled as ' 2 ' containing 1345 images. The dataset is split

Table 2 All the possible paths between the $S$ and $T$ from graph in Fig. 4 , ' $n$ ' is the number of hospitals covered in the path by the UAV

\begin{tabular}{lll}
\hline Path no. & All paths & {$[n$, cost $]$} \\
\hline 1. & $\mathrm{~S} \stackrel{3}{\rightarrow} H_{1} \stackrel{6}{\rightarrow} H_{2} \stackrel{6}{\rightarrow} \mathrm{T}$ & {$[2,15]$} \\
2. & $\mathrm{S} \stackrel{3}{\rightarrow} H_{1} \stackrel{8}{\rightarrow} H_{3} \stackrel{4}{\rightarrow} H_{2} \stackrel{6}{\rightarrow} \mathrm{T}$ & {$[3,21]$} \\
3. & $\mathrm{S} \stackrel{3}{\rightarrow} H_{1} \stackrel{8}{\rightarrow} H_{3} \stackrel{9}{\rightarrow} \mathrm{T}$ & {$[2,20]$} \\
4. & $\mathrm{~S} \stackrel{3}{\rightarrow} H_{1} \stackrel{\leftrightarrow}{\rightarrow} H_{2} \stackrel{4}{\rightarrow} H_{3} \stackrel{9}{\rightarrow} \mathrm{T}$ & {$[3,22]$} \\
5. & $\mathrm{~S} \stackrel{5}{\rightarrow} H_{3} \stackrel{9}{\rightarrow} \mathrm{T}$ & {$[1,14]$} \\
6. & $\mathrm{~S} \stackrel{5}{\rightarrow} H_{3} \stackrel{8}{\rightarrow} H_{1} \stackrel{6}{\rightarrow} H_{2} \stackrel{6}{\rightarrow} \mathrm{T}$ & {$[3,25]$} \\
7. & $\mathrm{~S} \stackrel{5}{\rightarrow} H_{3} \stackrel{4}{\rightarrow} H_{2} \stackrel{6}{\rightarrow} \mathrm{T}$ & {$[2,15]$} \\
\hline
\end{tabular}

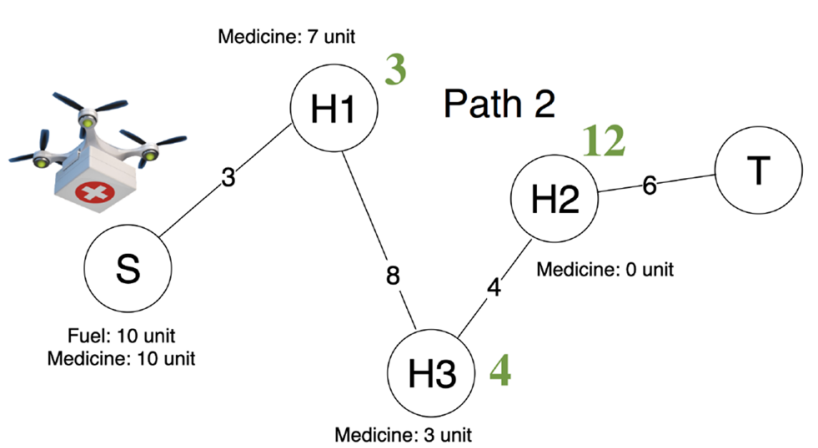

(a) Path number 2

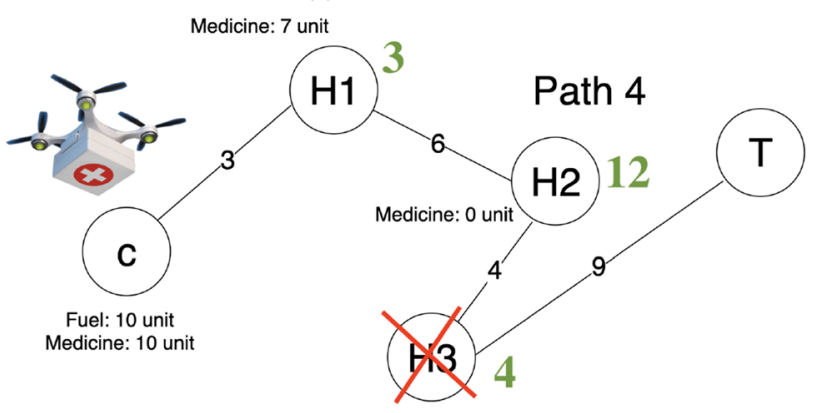

(b) Path number 4

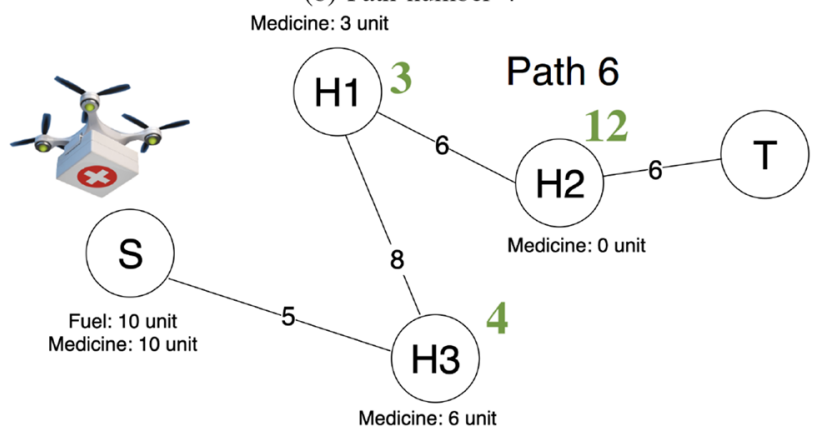

(c) Path number 6

Fig. 5 Candidate paths representing optimal paths

into training and testing sets. The testing part is set to be $25 \%$ of the whole dataset. The images in both the training and testing set are resized to $224 \times 224$ pixels. Table 3 shows the dataset distribution into the train and test set for the three classes.

Table 3 Dataset train-test split numbers of the three used classes

\begin{tabular}{llll}
\hline & & Classes & \\
\cline { 3 - 4 } Dataset & COVID-19 (0) & Normal (1) & Pneumonia (2) \\
\hline Train & 164 & 1005 & 1008 \\
Test & 55 & 336 & 337 \\
Total & 219 & 1341 & 1345 \\
\hline
\end{tabular}




\subsection{Evaluation metrics}

Following metrics are considered to evaluate the performance of the classifiers.

- Precision: represents the number of points from the actual positive class identified over all the identifications.

$$
\text { precision }=\frac{\text { true positives }(\mathrm{TP})}{\text { true positives }+ \text { false positives }(\mathrm{FP})} .
$$

- Recall: represents the number of points from the actual positive class identified over the total positive class points.

$$
\text { recall }=\frac{\text { true positives }(\mathrm{TP})}{\text { true positives }+ \text { false negatives }(\mathrm{FN})} .
$$

- $F_{1}$ score: $F_{1}$ score is a harmonic mean of precision and recall, which is needed to find a balance between the two evaluation metrics.

$$
\mathrm{F}_{1} \text { score }=\frac{2 * \text { precision } * \text { recall }}{\text { precision }+ \text { recall }} .
$$

- Accuracy: represents the proportion of points that are correctly classified over the total points.

$$
\text { dacc }=\frac{\mathrm{TP}+\mathrm{TN}}{\mathrm{TP}+\text { true negatives }(\mathrm{TN})+\mathrm{FP}+\mathrm{FN}} .
$$

- Area under ROC curve: As the area under a ROC curve represents the usefulness of a test in general, a greater area means a more useful test. The curve is plotted between the true-positive rate and the falsepositive rate.

\subsection{Analysis and comparison of proposed CNN model}

The experiments are performed on a chest X-ray dataset with three classes; COVID-19, normal, and pneumonia. The images in the dataset are pre-processed before feeding to the proposed model and other state-of-the-art models. The comparison of the proposed model is done with VGG-16 [44], ResNet50 [45], InceptionV3 [46], Xception [47], and InceptionResNetV2 [48]. We consider the test accuracy, test loss, classification metrics such as precision, recall, f1-score, and area under the ROC curve for comparison. In this section, we will discuss each of the mentioned metrics.

\subsubsection{Accuracy and loss}

Figure 6 shows the accuracy curve for both the training and test set over 50 epochs. All the six models have approximately an accuracy of around 90\%. In ResNet50 (Fig. 6b), the test set's accuracy is slightly greater than that of the train set. Rest classifiers have almost equal accuracy on average considering all 50 epochs. There is not much difference between all the classifiers' training loss values as shown in Fig. 7. Almost all the time, the loss was below 0.5 for the duration of 50 epochs. The curve between train and test loss for ResNet (Fig. 7b) is more or less the same.

\subsubsection{Confusion metrics}

Figure 8 shows the confusion matrix of all the six used classifiers, and the detailed analysis is done in Table 4. The proposed model shows the highest overall accuracy of $94.92 \%$ compared to the rest of the classifiers.

\subsubsection{Area under ROC curve}

The maximum area under the ROC curve for classes 0,1 , and 2 is shown by the proposed model (Fig. 9f) and the VGG-16 (Fig. 9a) model. The proposed model and the VGG-16 model show an almost similar area under the ROC curve for classes 0,1 , and 2 as $0.98,0.95$, and 0.95 . ResNet (Fig. 9b) is showing the least area under the ROC curve for classes 0,1 , and 2 as $0.95,0.91$, and 0.89 , respectively. The area under the micro and the macro averaging curve is a maximum of 0.96 for both the proposed model and the VGG-16 model, and its least value is 0.92 for the ResNet model.

\subsection{Analysis of the proposed path planning scheme}

The proposed path-planning scheme considers two factors: the onboard fuel presence and the medical kits' necessity by any hospital. Considering these two factors, the UAV's objective is to visit the maximum number of hospitals in the provided area. Table 5 shows all the possible paths that are possible from the given example as shown in Fig. 4. There are three scenarios as follows.

(1) if $(n=1)$ : UAV visits only one hospital before going to the target node. The total number of combinations will be ${ }^{3} C_{1} \times 1 !=3$.

(2) if $(n=2)$ : UAV visits two hospitals before going to the target node. The total number of combinations will be ${ }^{3} C_{2} \times 2 !=6$.

(3) if $(n=3)$ : UAV visits all the hospitals before going to the target node. The total number of combinations will be ${ }^{3} C_{3} \times 3 !=6$. 


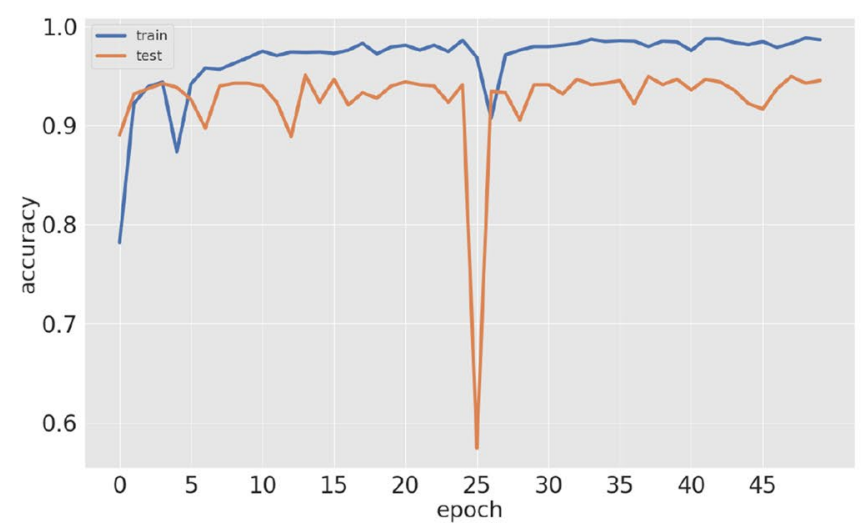

(a) VGG16

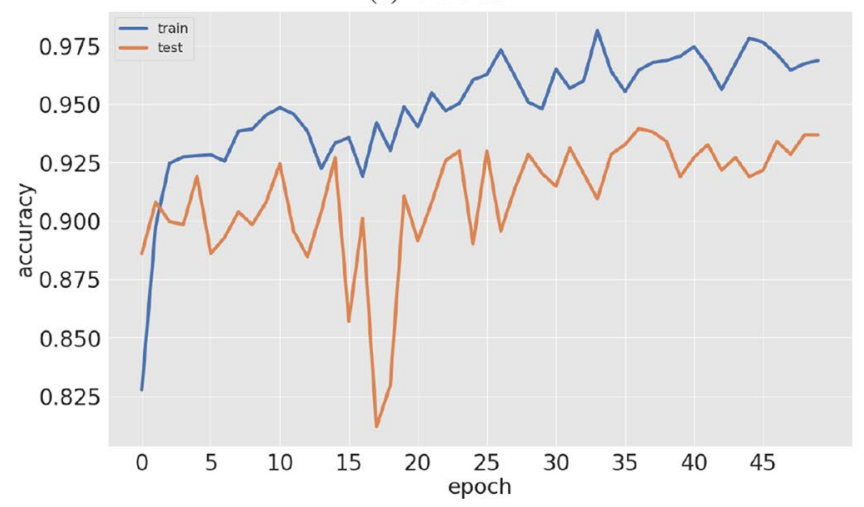

(c) Xception

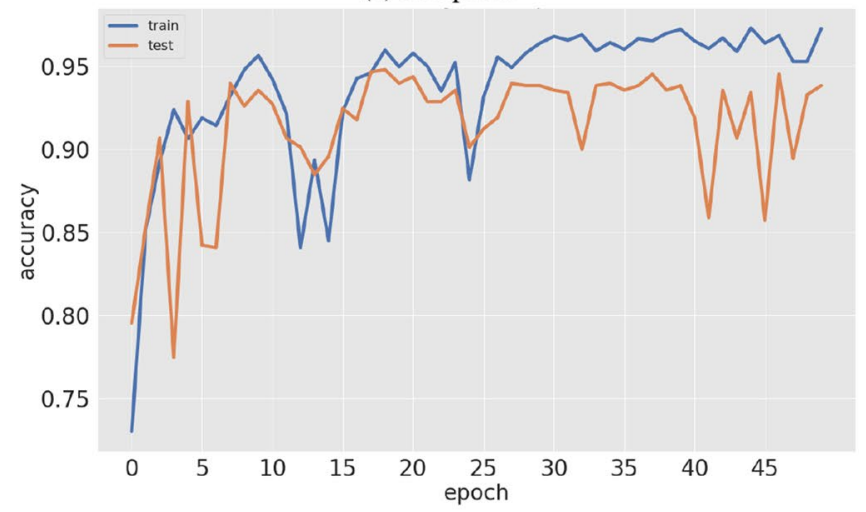

(e) InceptionResNetV2

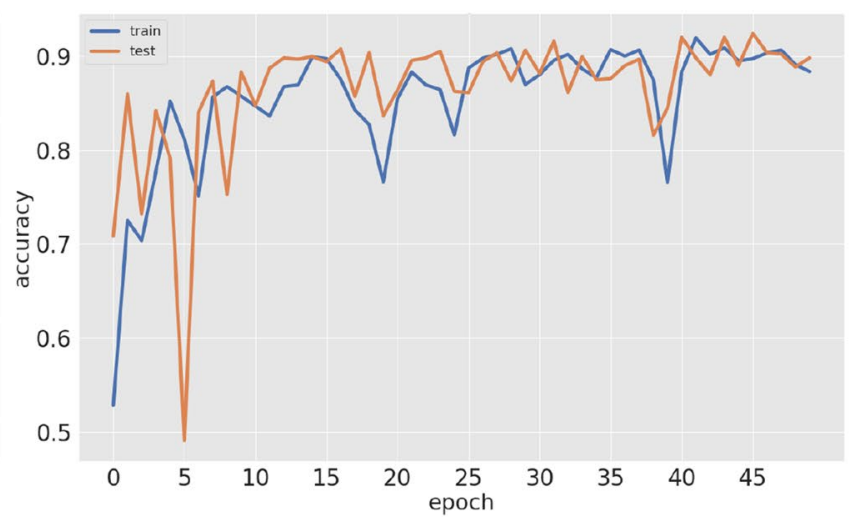

(b) ResNet50

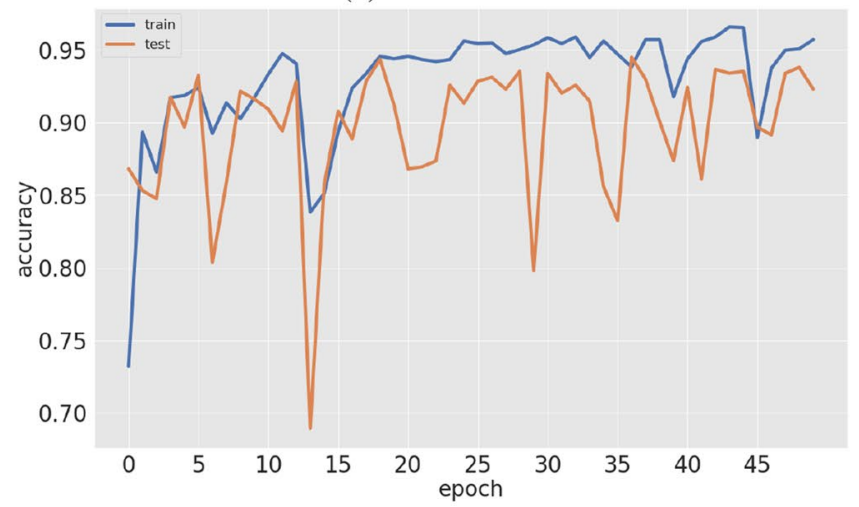

(d) InceptionV3

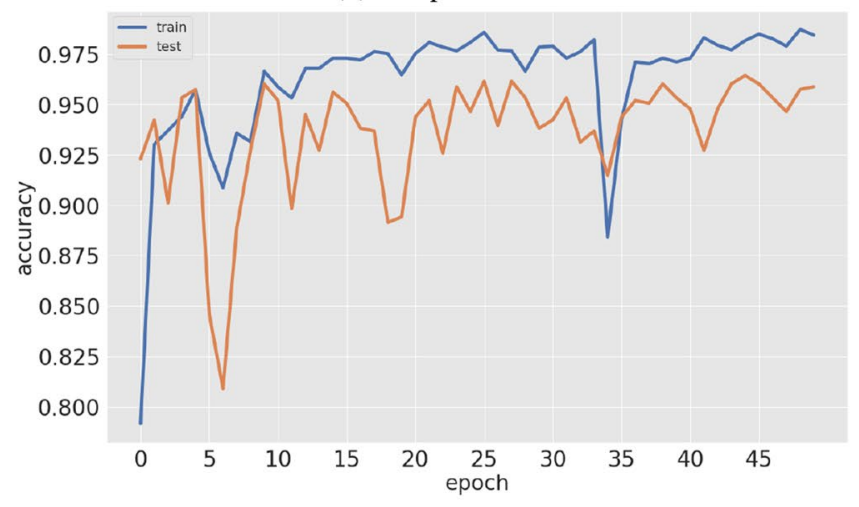

(f) Proposed Model

Fig. 6 Comparison of accuracy curve between the proposed model and the state-of-the-art models (blue and orange are the train and test curve, respectively)

Therefore the total number of cases will be fifteen. Out of these fifteen cases, few will be discarded based on the two factors that we have considered. If the path's cost is greater than the fuel in hand, then, in that case, UAV will never be able to reach the target; hence that path will be rejected. Due to this constraint, path numbers 1, 2, 5, 7, 9, 11, 13, and 15 are discarded as shown in Table 5. Considering the second constraint (UAV must have some medicine left in hand before visiting the next hospital), path numbers 5,8 , $10,11,13$, and 15 are discarded. After removing the paths based on the constraints, we are only left with path numbers $3,4,6,9,12$, and 14. Moreover, out of these paths, the path which visits all the three hospitals are path numbers 12 and 14, as shown in Table 5. Further, the minimum time taken is by path number 14 , which is also provided by our described proposed scheme as shown in Fig. 5 a. 


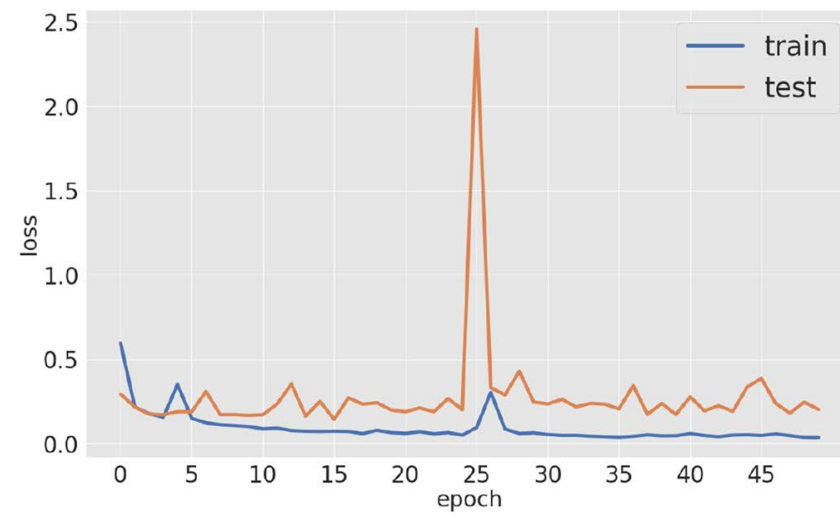

(a) VGG16

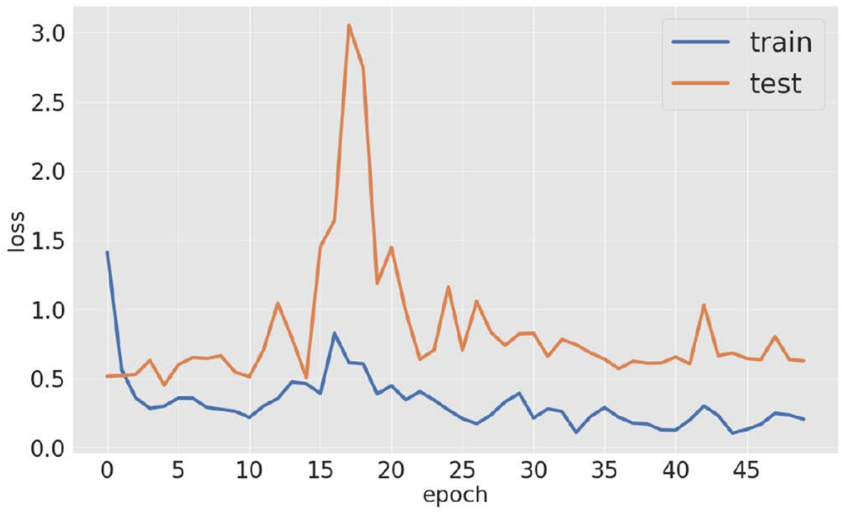

(c) Xception

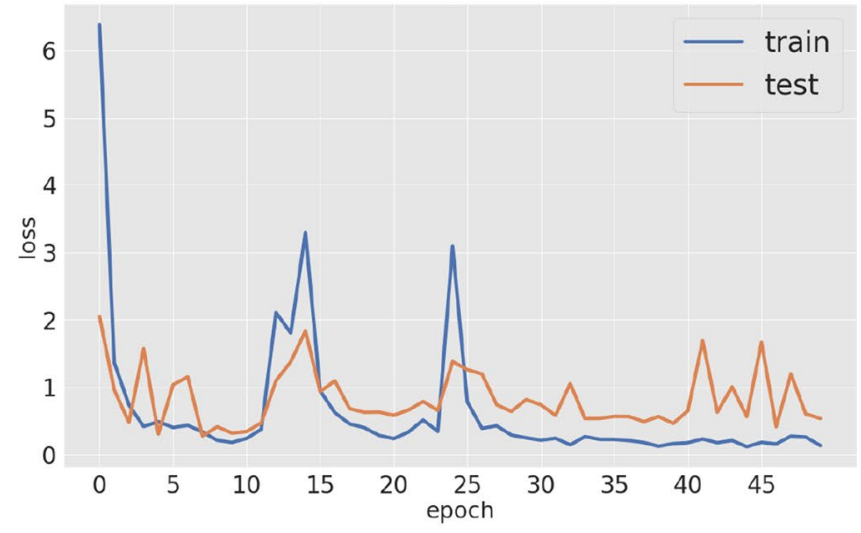

(e) InceptionResNetV2

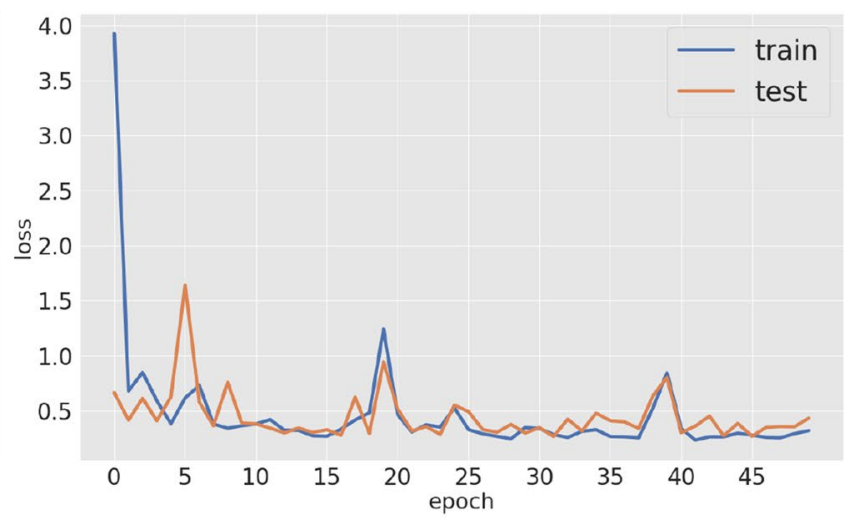

(b) ResNet50

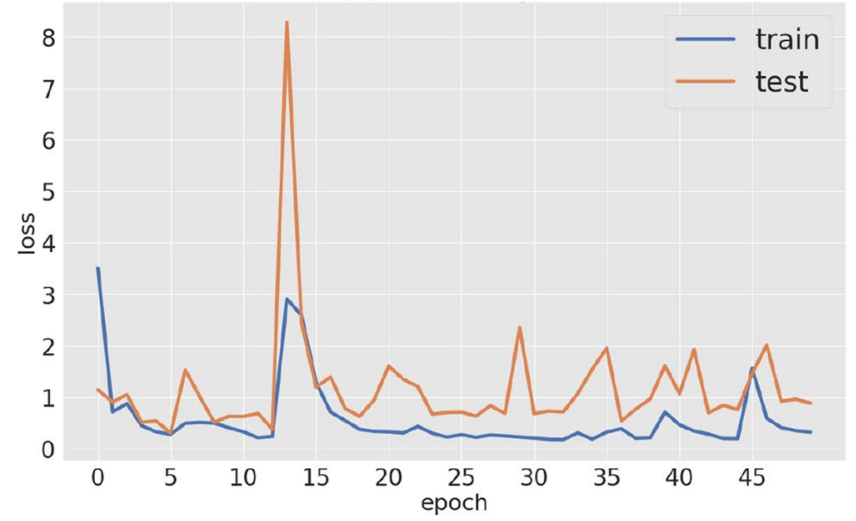

(d) InceptionV3

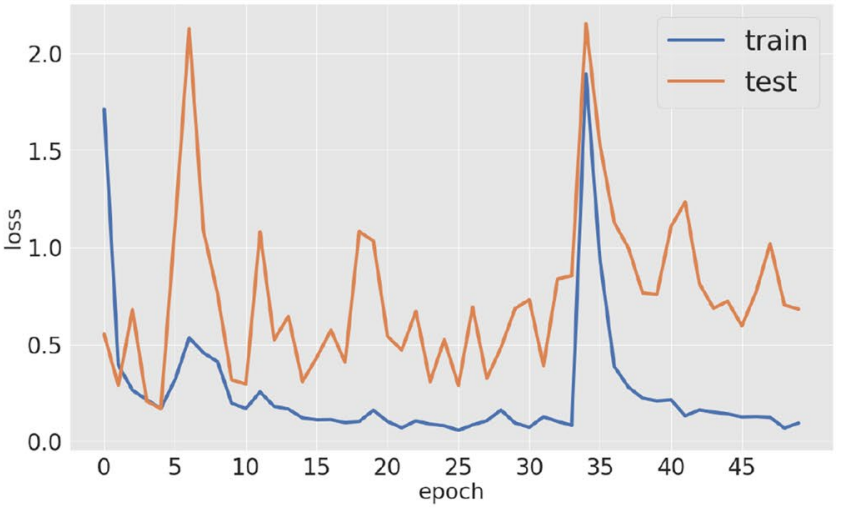

(f) Proposed Model

Fig. 7 Comparison of loss curve between the proposed model and the state-of-the-art models (blue and orange are the train and test curve, respectively)

\section{Conclusion and future directions}

Drones can gather real-time data to deliver payloads and have initiated the rapid evolution of many industrial, commercial, and recreational applications. Just as the mobile phone provided developing countries to leapfrog technology in communication, the delivery drone will lead to the same effect on the traditional transportation system. In the case of an emergency, inaccessible roads and traffics will no longer limit the delivery of blood, organs, medications, or other healthcare items. This article provides an optimal pathplanning scheme for delivering the emergency medical kit 


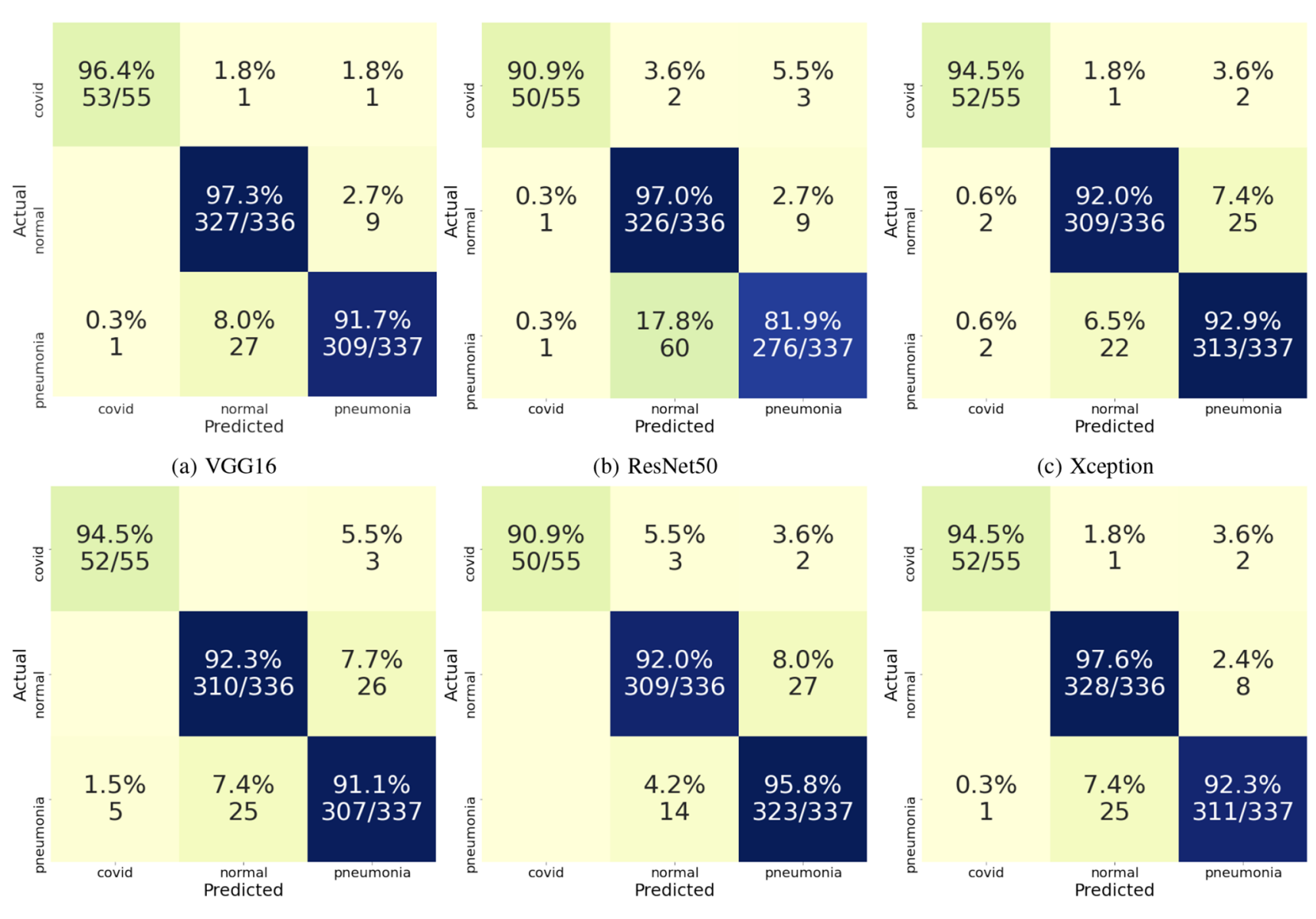

(d) InceptionV3

(e) InceptionResNetV2

(f) Proposed Model

Fig. 8 Comparison based on Confusion Matrix between the proposed model and the state-of-the-art models

in the COVID-19 pandemic using drones. In this paper, we developed and tested a deep-learning CNN model based on transfer learning to predict the possibility of COVID-19 in a chest X-ray image. For getting better results, the input chest X-ray dataset is pre-processed with image enhancement techniques. The UAVs encounter difficulties such as federal legislation, climate zones and topography, licensure and financial concerns, and social stands and acceptance in different countries. These obstacles need to be handled by people associated with the medical and drone industry, manufacturers, insurance firms, legislative officials, and government organizations. Although there are many benefits to drone technology in our daily lives; however, an equal number of difficulties are encountered. Finally, for this technology to succeed worldwide, the stakeholders must work collectively to blend it seamlessly into our societies. We can improve the dataset by generating synthetic images to increase the training performance, as the medical imaging data is limited and private. While training the network, we can additionally try various loss functions to see which loss function achieves better performance. 
Table 4 Comparison between the proposed model and the state-of-the-art model based on the classification metrics

\begin{tabular}{|c|c|c|c|c|c|c|c|c|c|}
\hline Model & Class & GT & Pred & Acc & Pre & $\operatorname{Rec}$ & F1 & Acc_overall & TP \\
\hline \multirow[t]{3}{*}{ VGG16 } & 0 & 55 & 54 & 99.59 & 0.98 & 0.96 & 0.97 & \multirow[t]{3}{*}{94.64} & \multirow[t]{3}{*}{689} \\
\hline & 1 & 336 & 355 & 94.92 & 0.92 & 0.97 & 0.95 & & \\
\hline & 2 & 337 & 319 & 94.78 & 0.97 & 0.92 & 0.94 & & \\
\hline \multirow[t]{3}{*}{ ResNet50 } & 0 & 55 & 52 & 99.04 & 0.96 & 0.91 & 0.93 & \multirow[t]{3}{*}{89.56} & \multirow[t]{3}{*}{652} \\
\hline & 1 & 336 & 388 & 90.11 & 0.84 & 0.97 & 0.90 & & \\
\hline & 2 & 337 & 288 & 89.97 & 0.96 & 0.82 & 0.88 & & \\
\hline \multirow[t]{3}{*}{ Xception } & 0 & 55 & 56 & 99.04 & 0.93 & 0.95 & 0.94 & \multirow[t]{3}{*}{92.58} & \multirow[t]{3}{*}{674} \\
\hline & 1 & 336 & 332 & 93.13 & 0.93 & 0.92 & 0.93 & & \\
\hline & 2 & 337 & 340 & 92.99 & 0.92 & 0.93 & 0.92 & & \\
\hline \multirow[t]{3}{*}{ InceptionV3 } & 0 & 55 & 57 & 98.91 & 0.91 & 0.95 & 0.93 & \multirow[t]{3}{*}{91.9} & \multirow[t]{3}{*}{669} \\
\hline & 1 & 336 & 335 & 92.99 & 0.93 & 0.92 & 0.92 & & \\
\hline & 2 & 337 & 336 & 91.94 & 0.91 & 0.91 & 0.91 & & \\
\hline \multirow[t]{3}{*}{ InceptionResNetV2 } & 0 & 55 & 50 & 99.31 & 1.00 & 0.91 & 0.95 & \multirow[t]{3}{*}{93.68} & \multirow[t]{3}{*}{682} \\
\hline & 1 & 336 & 326 & 93.96 & 0.95 & 0.92 & 0.93 & & \\
\hline & 2 & 337 & 352 & 94.09 & 0.92 & 0.96 & 0.94 & & \\
\hline \multirow{3}{*}{$\begin{array}{l}\text { Proposed Model } \\
\text { (without pre-processing) }\end{array}$} & 0 & 55 & 55 & 98.63 & 0.91 & 0.91 & 0.91 & \multirow[t]{3}{*}{93.96} & \multirow[t]{3}{*}{684} \\
\hline & 1 & 336 & 351 & 94.64 & 0.92 & 0.96 & 0.94 & & \\
\hline & 2 & 337 & 322 & 94.64 & 0.96 & 0.92 & 0.94 & & \\
\hline \multirow{3}{*}{$\begin{array}{l}\text { Proposed model } \\
\text { (with pre-processing) }\end{array}$} & 0 & 55 & 53 & 99.45 & 0.98 & 0.95 & 0.96 & \multirow[t]{3}{*}{94.92} & \multirow[t]{3}{*}{691} \\
\hline & 1 & 336 & 354 & 95.33 & 0.93 & 0.98 & 0.95 & & \\
\hline & 2 & 337 & 321 & 95.05 & 0.97 & 0.92 & 0.95 & & \\
\hline
\end{tabular}

GT Ground Truth, Pred Predicted values, Acc Accuracy, Pre Precision, Rec Recall, F1 F1-score, TP True positives)
The proposed approach does not substitute a medical diagnosis since further thorough research could be done with a larger dataset. Under those circumstances, the proposed approach will act as a tool to detect COVID-19 infected patients from chest X-rays more accurately and in stipulated time. Moreover, we will collaborate with health care practitioners to get executable data in a high count and with high-resolution images. In the future, we plan to use more X-ray images of patients with COVID-19 symptoms and update the InceptionV3 model using Federated
Learning that provides a distributed approach for training the model. With X-ray images of other lung-related diseases, we can generate a classifier having more than three classes, thus encouraging the proposed approach's efficiency. Further, we will deploy our model into a free online platform of image classification so that the hospitals and medical clinics available worldwide would be able to identify diseases using chest X-ray images without the need for building their specific classification platform. 


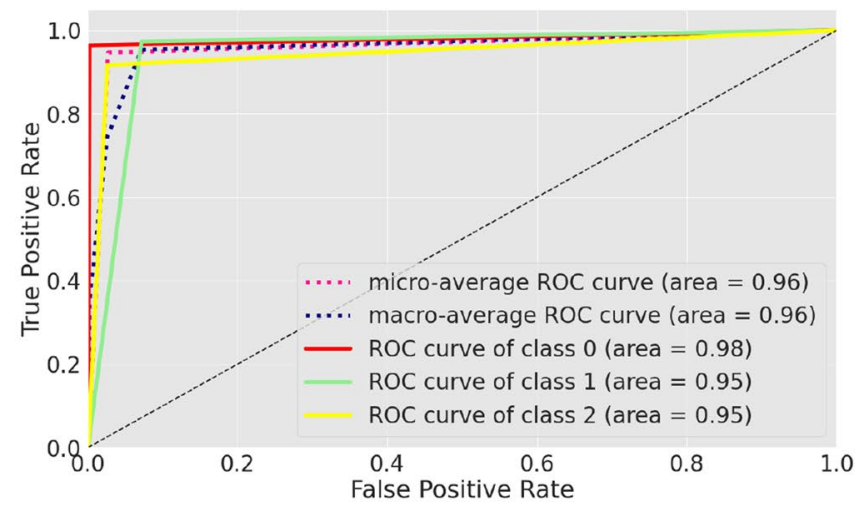

(a) VGG16

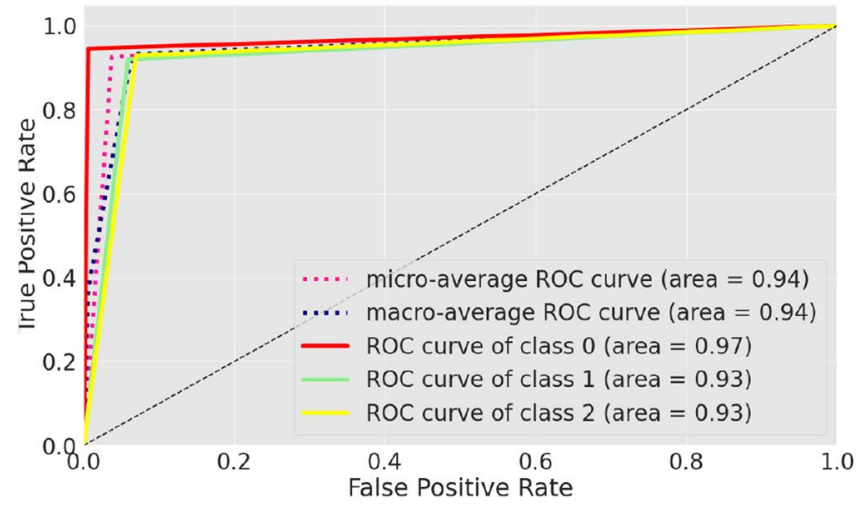

(c) Xception

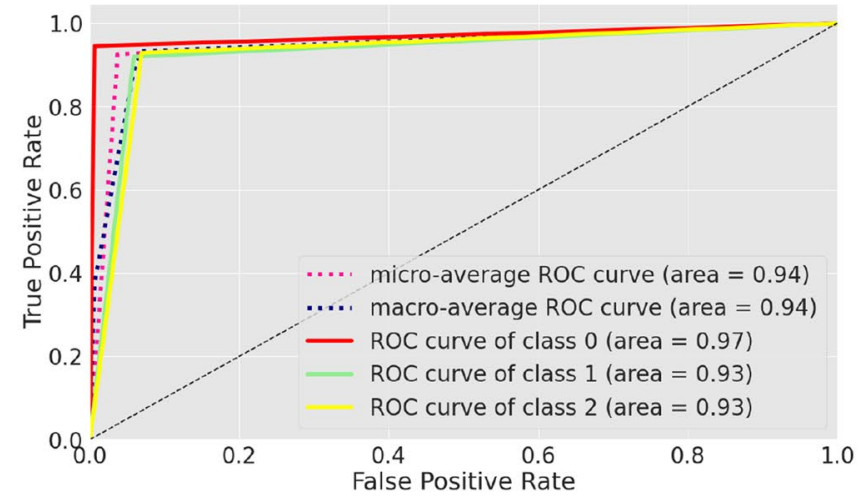

(e) InceptionResNetV2

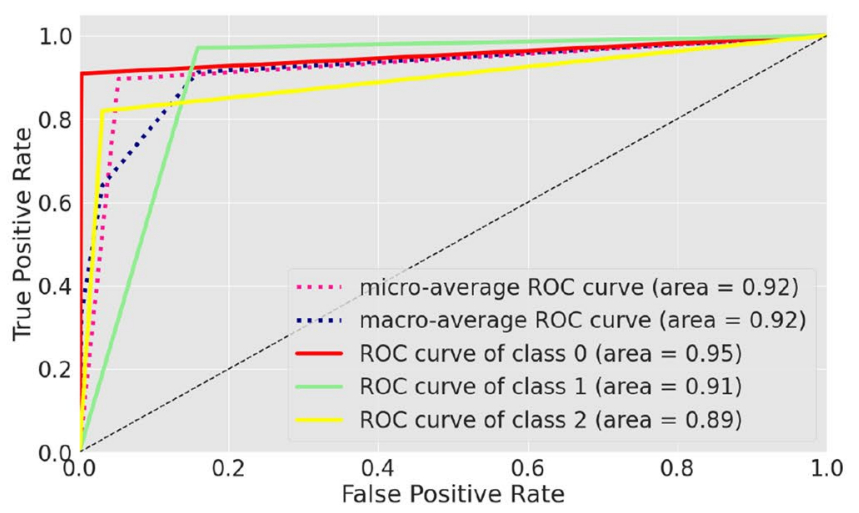

(b) ResNet50

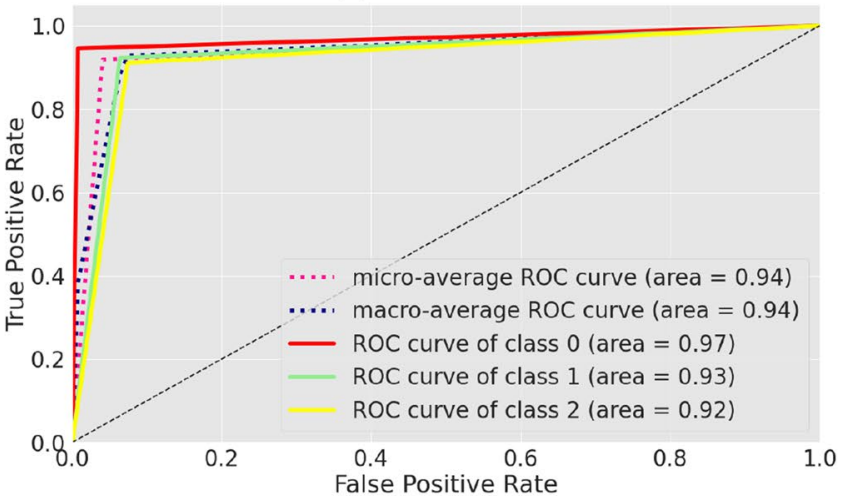

(d) InceptionV3

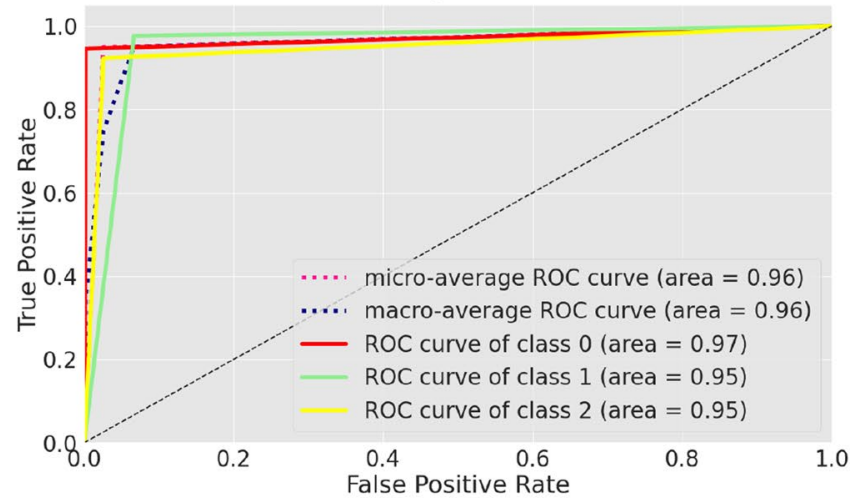

(f) Proposed Model

Fig. 9 Comparison of ROC curve between the proposed model and the state-of-the-art models 
Table 5 All the possible combinations of paths between the Source $(S)$ and Target $(T)$ from the graph in Fig. 4, ' $n$ ' is the number of hospitals covered in the path by the UAV, $(\checkmark)$ signifies that particular path fulfills the condition else it is marked as $(\boldsymbol{X})$

\begin{tabular}{|c|c|c|c|c|c|}
\hline Path. no. & $n$ & Path from $S$ to $T$ & $\begin{array}{l}\text { Feasible accord- } \\
\text { ing to fuel }\end{array}$ & $\begin{array}{l}\text { Feasible according } \\
\text { to medicine }\end{array}$ & Cost \\
\hline 1. & 1 & $\mathrm{~S} \stackrel{3}{\rightarrow} H_{1} \stackrel{17}{\longrightarrow} \mathrm{T}$ & $x$ & $\checkmark$ & - \\
\hline 2. & 1 & $\mathrm{~S} \stackrel{12}{\rightarrow} H_{2} \stackrel{6}{\rightarrow} \mathrm{T}$ & $x$ & $\checkmark$ & - \\
\hline 3. & 1 & $\mathrm{~S} \stackrel{5}{\rightarrow} H_{3} \stackrel{9}{\rightarrow} \mathrm{T}$ & $\checkmark$ & $\checkmark$ & 14 \\
\hline 4. & 2 & $\mathrm{~S} \stackrel{3}{\rightarrow} H_{1} \stackrel{6}{\rightarrow} H_{2} \stackrel{6}{\rightarrow} \mathrm{T}$ & $\checkmark$ & $\checkmark$ & 15 \\
\hline 5. & 2 & $\mathrm{~S} \stackrel{12}{\rightarrow} H_{2} \stackrel{4}{\rightarrow} H_{3} \stackrel{9}{\rightarrow} \mathrm{T}$ & $x$ & $x$ & - \\
\hline 6. & 2 & $\mathrm{~S} \stackrel{3}{\rightarrow} H_{1} \stackrel{8}{\rightarrow} H_{3} \stackrel{9}{\rightarrow} \mathrm{T}$ & $\checkmark$ & $\checkmark$ & 20 \\
\hline 7. & 2 & $\mathrm{~S} \stackrel{12}{\longrightarrow} H_{2} \stackrel{6}{\rightarrow} H_{1} \stackrel{17}{\longrightarrow} \mathrm{T}$ & $x$ & $\checkmark$ & - \\
\hline 8. & 2 & $\mathrm{~S} \stackrel{5}{\rightarrow} H_{3} \stackrel{4}{\rightarrow} H_{2} \stackrel{6}{\rightarrow} \mathrm{T}$ & $\checkmark$ & $x$ & - \\
\hline 9. & 2 & $\mathrm{~S} \stackrel{5}{\rightarrow} H_{3} \stackrel{8}{\rightarrow} H_{1} \stackrel{17}{\longrightarrow} \mathrm{T}$ & $x$ & $\checkmark$ & 30 \\
\hline 10. & 3 & $\mathrm{~S} \stackrel{3}{\rightarrow} H_{1} \stackrel{6}{\rightarrow} H_{2} \stackrel{4}{\rightarrow} H_{3} \stackrel{9}{\rightarrow} \mathrm{T}$ & $\checkmark$ & $x$ & - \\
\hline 11. & 3 & $\mathrm{~S} \stackrel{12}{\longrightarrow} H_{2} \stackrel{4}{\rightarrow} H_{3} \stackrel{8}{\rightarrow} H_{1} \stackrel{17}{\longrightarrow} \mathrm{T}$ & $x$ & $x$ & - \\
\hline 12. & 3 & $\mathrm{~S} \stackrel{5}{\rightarrow} H_{3} \stackrel{8}{\rightarrow} H_{1} \stackrel{6}{\rightarrow} H_{2} \stackrel{6}{\rightarrow} \mathrm{T}$ & $\checkmark$ & $\checkmark$ & 25 \\
\hline 13. & 3 & $\mathrm{~S} \stackrel{5}{\rightarrow} H_{3} \stackrel{4}{\rightarrow} H_{2} \stackrel{6}{\rightarrow} H_{1} \stackrel{17}{\rightarrow} \mathrm{T}$ & $x$ & $x$ & - \\
\hline 14. & 3 & $\mathrm{~S} \stackrel{3}{\rightarrow} H_{1} \stackrel{8}{\rightarrow} H_{3} \stackrel{4}{\rightarrow} H_{2} \stackrel{6}{\rightarrow} \mathrm{T}$ & $\checkmark$ & $\checkmark$ & 21 \\
\hline 15. & 3 & $\mathrm{~S} \stackrel{12}{\rightarrow} H_{2} \stackrel{6}{\rightarrow} H_{1} \stackrel{8}{\rightarrow} H_{3} \stackrel{9}{\rightarrow} \mathrm{T}$ & $x$ & $x$ & - \\
\hline
\end{tabular}

Acknowledgements This project was funded by the Deanship of Scientific Research (DSR) at King Abdulaziz University, Jeddah, under grant no. RG-23-611-38. The authors, therefore, acknowledge with thanks DSR for technical and financial support.

\section{References}

1. Choi-Fitzpatrick, A., Chavarria, D., Cychosz, E., Dingens, J.P, Duffey, M., Koebel, K., Siriphanh, S., Yurika Tulen, M., Watanabe, H., Juskauskas. T. et al.: Up in the air: a global estimate of non-violent drone use 2009-2015 (2016)

2. Chhikara, P., Tekchandani, R., Kumar, N., Chamola, V., Guizani, M.: Dcnn-ga: a deep neural net architecture for navigation of uav in indoor environment. IEEE Internet Things J. 8(6), 4448-4460 (2021)

3. Bamburry, D.: Drones: designed for product delivery. Des Manag Rev 26(1), 40-48 (2015)

4. Nesta.: Flying high: the future of drone technology in UK cities. https://www.nesta.org.uk/report/flying-high-challenge-future-ofdrone-technology-in-uk-cities/ (2020)

5. Chhikara, P., Tekchandani, R., Kumar, N., Guizani, M., Hassan, M.M.: Federated learning and autonomous UAVs for hazardous zone detection and AQI prediction in IoT environment. IEEE Internet Things J. (2021)

6. Santhanam, V.: How drones could change the future of healthcare delivery. https://www.weforum.org/agenda/2020/05/medic al-drone-delivery-india-africa-modernize-last-mile/ (2020)

7. Ugalmugle, S.: Medical drones market size by application (blood transfer, drugs/pharmaceutical transfer, vaccination program), end-use (emergency medical services, blood banks), industry analysis report, country outlook, application potential, competitive market share and forecast, 2019-2025. https://www.gminsights. com/industry-analysis/medical-drones-market (2021)
8. Cohn, M.: University of Maryland medical center transplants first drone-delivered organ. https://www.baltimoresun.com/health/bshs-organ-delivered-by-drone-20190426-story.html (2020)

9. Hii, M.S.Y., Courtney, P., Royall, P.G.: An evaluation of the delivery of medicines using drones. Drones 3(3), 52 (2019)

10. Barnawi, A., Chhikara, P., Tekchandani, R., Kumar, N., Alzahrani, B.: Artificial intelligence-enabled internet of things-based system for COVID-19 screening using aerial thermal imaging. Future Gener. Comput Syst FGCS 124, 119-132 (2021)

11. Chamola, V., Hassija, V., Gupta, V., Guizani, M.: A comprehensive review of the COVID-19 pandemic and the role of IoT, drones, AI, blockchain, and $5 \mathrm{G}$ in managing its impact. IEEE Access 8, 90 225-90 265 (2020)

12. D. of Information and I. Public Relations, Punjab. Punjab govt to provide 50000 free covid care kits to active patients in hospitals and homes. http://diprpunjab.gov.in/?q=content/punjabgovt-provide-50000-free-covid-care-kits-active-patients-hospi tals-homes (2021)

13. Ayamga, M., Akaba, S., Nyaaba, A.A.: Multifaceted applicability of drones: a review. Technol. Forecast. Soc. Change 167, 120677 (2021)

14. Food, U., Administration, D. et al.: Accelerated emergency use authorization (eua) summary covid-19 rt-pcr test (laboratory corporation of America)

15. Sekeroglu, B., Ozsahin, I.: Detection of covid-19 from chest x-ray images using convolutional neural networks. SLAS Technol: Transl Life Sci Innov 25(6), 553-565 (2020)

16. Rubin, G.D., Ryerson, C.J., Haramati, L.B., Sverzellati, N., Kanne, J.P., Raoof, S., Schluger, N.W., Volpi, A., Yim, J.-J., Martin, I.B., et al.: The role of chest imaging in patient management during the covid-19 pandemic: a multinational consensus statement from the fleischner society. Chest 158(1), 106-116 (2020)

17. Minaee, S., Kafieh, R., Sonka, M., Yazdani, S., Soufi, G.J.: Deepcovid: predicting covid-19 from chest X-ray images using deep transfer learning. Med Image Anal 65, 101794 (2020) 
18. Shi, W., Li, J., Xu, W., Zhou, H., Zhang, N., Zhang, S., Shen, X.: Multiple drone-cell deployment analyses and optimization in drone assisted radio access networks. IEEE Access 6, 12 518-12 529 (2018)

19. Sharma, V., You, I., Pau, G., Collotta, M., Lim, J.D., Kim, J.N.: Lorawan-based energy-efficient surveillance by drones for intelligent transportation systems. Energies 11(3), 573 (2018)

20. Shakhatreh, H., Khreishah, A., Alsarhan, A., Khalil, I., Sawalmeh, A., Othman, N.S.: Efficient 3D placement of a UAV using particle swarm optimization, In: 2017 8th international conference on information and communication systems (ICICS). IEEE, pp. 258-263 (2017)

21. Ghamry, K.A., Kamel, M.A., Zhang, Y.: Multiple UAVs in forest fire fighting mission using particle swarm optimization, In: 2017 International conference on unmanned aircraft systems (ICUAS). IEEE, pp. 1404-1409 (2017)

22. Berger, C., Wzorek, M., Kvarnström, J., Conte, G., Doherty, P., Eriksson, A.: Area coverage with heterogeneous uavs using scan patterns, In: IEEE international symposium on safety, security, and rescue robotics (SSRR). IEEE 2016, 342-349 (2016)

23. You, I., Sharma, V., Atiquzzaman, M., Choo, K.-K.R.: GDTN: Genome-based delay tolerant network formation in heterogeneous 5G using inter-UA collaboration. PloS One 11(12), e0167913 (2016)

24. Ismael, A.M., Şengür, A.: Deep learning approaches for COVID19 detection based on chest X-ray images. Expert Syst Appl. 164, 114054 (2021)

25. Jain, R., Gupta, M., Taneja, S., Hemanth, D.J.: Deep learning based detection and analysis of COVID-19 on chest X-ray images. Appl. Intell. 51(3), 1690-1700 (2021)

26. Ibrahim, A.U., Ozsoz, M., Serte, S., Al-Turjman, F., Yakoi, P.S.: Pneumonia classification using deep learning from chest X-ray images during COVID-19. Cogn. Comput, pp. 1-13 (2021)

27. Wang, Z., Xiao, Y., Li, Y., Zhang, J., Lu, F., Hou, M., Liu, X.: Automatically discriminating and localizing COVID-19 from community-acquired pneumonia on chest X-rays. Pattern Recognit 110, 107613 (2021)

28. Mahmud, T., Rahman, M.A., Fattah, S.A.: CovXNet: a multidilation convolutional neural network for automatic COVID-19 and other pneumonia detection from chest $\mathrm{x}$-ray images with transferable multi-receptive feature optimization. Comput. Biol. Med. 122, 103869 (2020)

29. Hassantabar, S., Ahmadi, M., Sharifi, A.: Diagnosis and detection of infected tissue of COVID-19 patients based on lung X-ray image using convolutional neural network approaches. Chaos Solitons Fractals 140, 110170 (2020)

30. Islam, M.Z., Islam, M.M., Asraf, A.: A combined deep CNNLSTM network for the detection of novel coronavirus (COVID19) using X-ray images. Informatics in Medicine Unlocked, p. $100412(2020)$

31. Shibly, K.H., Dey, S.K., Islam, M.T.U., Rahman, M.M.: COVID faster R-CNN: a novel framework to diagnose novel coronavirus disease (COVID-19) in X-ray images. medRxiv (2020)

32. Abraham, B., Nair, M.S.: Computer-aided detection of COVID19 from X-ray images using multi-CNN and Bayesnet classifier. Biocybern. Biomed. Eng. (2020)

33. Vrbančič, G., Pečnik, Š., Podgorelec, V.: Identification of COVID19 X-ray images using CNN with optimized tuning of transfer learning, In: 2020 international conference on innovations in intelligent systems and applications (INISTA)

34. Fathurahman, M., Fauzi, S.C., Haryanti, S.C., Rahmawati, U.A., Suherlan, E.: Implementation of 1D-convolution neural network for pneumonia classification based chest X-ray image, In: International conference on soft computing and data mining. Springer, pp. 181-191 (2020)

35. Toraman, S., Alakus, T.B., Turkoglu, I.: Convolutional capsnet: a novel artificial neural network approach to detect COVID-19 disease from X-ray images using capsule networks. Chaos Solitons Fractals 140, 110122 (2020)

36. Chen, X.: Image enhancement effect on the performance of convolutional neural networks (2019)

37. Chhikara, P., Singh, P., Gupta, P., Bhatia, T.: Deep convolutional neural network with transfer learning for detecting pneumonia on chest x-rays. In: Advances in bioinformatics, multimedia, and electronics circuits and signals. Springer, Berlin pp. 155-168 (2020)

38. Kuflinski, Y.: How medical image analysis will benefit patients and physicians. https://www.iflexion.com/blog/medical-imageanalysis (2021)

39. Van Vliet, L.J., Young, I.T., Beckers, G.L.: A nonlinear Laplace operator as edge detector in noisy images. Comput. Vis. Graph. Image Process. 45(2), 167-195 (1989)

40. Kanopoulos, N., Vasanthavada, N., Baker, R.L.: Design of an image edge detection filter using the Sobel operator. IEEE J. Solid-state Circuits 23(2), 358-367 (1988)

41. Hummel, R.A., Kimia, B., Zucker, S.W.: Deblurring gaussian blur. Comput. Vis. Graphi. Image Process. 38(1), 66-80 (1987)

42. Reza, A.M.: Realization of the contrast limited adaptive histogram equalization (CLAHE) for real-time image enhancement. J. VLSI Signal Process. Syst. Signal Image Video Technol. 38(1), 35-44 (2004)

43. Chowdhury, M.E.H., Rahman, T., Khandakar, A., Mazhar, R., Kadir, M.A., Mahbub, Z.B., Islam, K.R., Khan, M.S., Iqbal, A., Emadi, N.A., Reaz, M.B.I., Islam, M.T.: Can ai help in screening viral and covid-19 pneumonia? IEEE Access 8, 132 665-132 676 (2020)

44. Simonyan, K., Zisserman, A.: Very deep convolutional networks for large-scale image recognition, arXiv preprint arXiv:1409.1556 (2014)

45. He, K., Zhang, X., Ren, S., Sun, J.: Deep residual learning for image recognition. In: Proceedings of the IEEE conference on computer vision and pattern recognition, pp. 770-778 (2016)

46. Szegedy, C., Ioffe, S., Vanhoucke, V., Alemi, A.: Inception-v4, inception-resnet and the impact of residual connections on learning. arXiv preprint arXiv:1602.07261 (2016)

47. Chollet, F.: Xception: Deep learning with depthwise separable convolutions. In: Proceedings of the IEEE conference on computer vision and pattern recognition. pp. 1251-1258 (2017)

48. Szegedy, C., Ioffe, S., Vanhoucke, V., Alemi, A.: Inception-v4, inception-resnet and the impact of residual connections on learning (2016)

Publisher's Note Springer Nature remains neutral with regard to jurisdictional claims in published maps and institutional affiliations. 